\title{
Does PROM Always Reflect the Reality? Our Experience with the Olerud-Molander Ankle Score (OMAS) in the Ongoing Ankle Fracture p-RCT (DoWeCAST)
}

\author{
Ramy Khojaly*1,2, Gozie Offiah ${ }^{3}$, Ruairi MacNiocaill ${ }^{2}$, Claire Condron ${ }^{3}$, Arnold Hill ${ }^{3}$ and May Cleary ${ }^{2,4}$ \\ ${ }^{1}$ Department of Surgery, Orthopaedic surgery, clinical lecturer, Royal College of Surgeons in Ireland, Ireland \\ ${ }^{2}$ Department of Orthopaedic surgery, University Hospital Waterford, Ireland \\ ${ }^{3}$ Department of Surgery, Royal College of Surgeons in Ireland, Ireland \\ ${ }^{4}$ Department of Orthopaedic surgery, University College Cork, Ireland
}

*Corresponding author: Ramy Khojaly, Orthopaedic clinical lecturer, Department of Surgery, Royal College of Surgeons in Ireland, Ireland.

Received Date: January 20, 2020

Published Date: January 31,2020

\section{Introduction}

The use of Patient-Reported Outcome Measures (PROMs) has become more prevalent in recent years. PROMs provide a direct reflection of the patient's health and function to the effect of treatment or intervention provided [1-3]. The Olerud-Molander Ankle Score (OMAS) is a commonly used PROM in ankle fracture research and often, it is the primary outcome measure [4-10]. This questionnaire was developed in 1984, specifically, to evaluate the function of patients with ankle fractures [11]. A recent systematic review evaluating patients reported outcome measures used for adults with ankle fracture, found that OMAS has sufficient levels of reliability, internal consistency and construct validity. However, the review did not recommend any particular PROM due to insufficient supporting evidence [12].

(DoWeCAST) trial is a pragmatic randomized controlled multi-center trial, comparing Immediate Weight-Bearing (IWB) in a walking boot versus Non-Weight Bearing (NWB) and immobilization in a cast, following ORIF of all types of unstable adult's ankle fractures [13]. The trial which started in January 2019 is forecast to be the largest and the most comprehensive trial investigating the immediate post-operative weight-bearing of ankle fractures worldwide and is expected to complete enrollment by July 2020 .

\section{Discussion}

In this article, we share some of our experiences of using OMAS as a primary outcome measure in trials assessing weight-bearing following ankle fracture fixation. The OMAS ranges from 0 to 100 with 100 representing the normal ankle function. The questionnaire has nine questions, assessing pain (25 points), stiffness (10 points), swelling (10 points), stair climbing (10 points), running (5 points), jumping ( 5 points), squatting ( 5 points), support (10 points) and activity of daily life (20 points).

Pain is an important component of the OMAS questionnaire and as such is assigned the highest points of 25. The patients chose out of the six options. If the patient has no pain, he will score 25 out of 25 , if he does have pain while walking on even or uneven surface or indoor, he will score $20,10,5$ respectively. If the pain is consistent and severe, the score is zero. Most of the patients in the NWB group are pain-free at six weeks as they are in cast and have not started to weight-bear yet, while patients in the IWB group come to the six weeks assessment walking, often, in their regular shoes without the walking boot. It is expected that some pain might still be present at 6 weeks postoperatively.

It is quite apparent that a patient coming to the clinic walking full weight-bearing is functionally better than patients walking non- 
weight bearing with two crutches, but this is not reflected by OMAS. Stiffness and swelling are variables with no clear assessment tool to facilitate differentiation in scale. While all patients with ankle fractures are expected to have swelling and stiffness for several months, some patients might have less swelling and stiffness than others, but the OMAS is unable to detect that difference especially in the early few months after surgery. Running, jumping, and squatting are individual-related activities and many patients do not regularly participate in these activities, especially elderly patients. These items is frequently unanswered which markedly affects the calculation of the final score. Some elderly patients are already dependent on a walking aid even before the injury to the ankle. Their ankle fracture is not necessarily the reason for them to continue to be dependent on a walking aid. OMAS does not consider the patient status prior to the injury.

Ability and willingness to return to work after surgery has many dependent factors, for example, age, work type, sick benefits and others. A patient who is unwilling to return to work for any of these reasons, will less likely to select the option "same as before injury", (score 20 points) independent of their ankle condition.

\section{Conclusion}

Investigators and surgeons must keep in mind the weakness in OMAS score for assessing the efficacy of weight-bearing following ankle fracture fixation. A more reliable PROM targeted to this group of patients is needed and hence the initiation of the randomized controlled multi-center trial by our group.

\section{Acknowledgement}

None.

\section{Conflict of Interest}

No conflict of interest.

\section{References}

1. Weldring T, Smith SMS (2013) Patient-Reported Outcomes (PROs) and Patient-Reported Outcome Measures (PROMs). Health Serv Insights 6: 61-68.
2. Vodicka E, Kim K, Devine EB, Gnanasakthy A, Scoggins JF, et al. (2015) Inclusion of patient-reported outcome measures in registered clinical trials: Evidence from ClinicalTrials.gov (2007-2013). Contemp Clin Trials 43: 1-9.

3. Dawson J, Doll H, Fitzpatrick R, Jenkinson C, Carr AJ (2010) The routine use of patient reported outcome measures in healthcare settings. BMJ 340: c186.

4. Dehghan N, McKee MD, Jenkinson RJ, Schemitsch EH, Stas V, et al. (2016) Early Weightbearing and Range of Motion Versus Non-Weightbearing and Immobilization After Open Reduction and Internal Fixation of Unstable Ankle Fractures: A Randomized Controlled Trial. J Orthop Trauma 30(7): 345-352.

5. Black JDJ, Bhavikatti M, Al Hadithy N, Hakmi A, Kitson J (2013) Early weight-bearing in operatively fixed ankle fractures: a systematic review. Foot (Edinb) 23(2-3): 78-85.

6. Smeeing DPJ, Houwert RM, Briet JP, Kelder JC, Segers MJM, et al. (2015) Weight-Bearing and Mobilization in the Postoperative Care of Ankle Fractures: A Systematic Review and Meta-Analysis of Randomized Controlled Trials and Cohort Studies. PLoS One 10(2): e0118320.

7. Vioreanu M, Dudeney S, Hurson B, Kelly E, O Rourke K, et al. (2007) Early mobilization in a removable cast compared with immobilization in a cast after operative treatment of ankle fractures: a prospective randomize study. Foot Ankle Int 28(1): 13-9.

8. Van Laarhoven CJ, Meeuwis JD, van der Werken C (1996) Postoperative treatment of internally fixed ankle fractures: a prospective randomised study. J Bone Joint Surg Br 78(3): 395-399.

9. Gul A, Batra S, Mehmood S, Gillham N (2009) Immediate unprotected weight-bearing of operatively treated ankle fractures. Acta Orthop Belg 73(3): 360-365.

10. Smeeing DPJ, Houwert RM, Briet JP, Groenwold RHH, Lansink KWW, et al. (2018) Weight-bearing or non-weight-bearing after surgical treatment of ankle fractures: a multicenter randomized controlled trial. Eur J Trauma Emerg Surg 24.

11. Olerud C, Molander H (1984) A scoring scale for symptom evaluation after ankle fracture. Arch Orthop Trauma Surg 103(3): 190-194.

12. McKeown R, Ellard DR, Rabiu AR, Karasouli E, Kearney RS (2019) A systematic review of the measurement properties of patient reported outcome measures used for adults with an ankle fracture. J Patient Rep Outcomes 3(1): 70.

13. (2020) ISRCTN-ISRCTN76410775: Do we have to keep patients in a cast and prevent them from weight bearing after surgical repair of ankle fractures? 